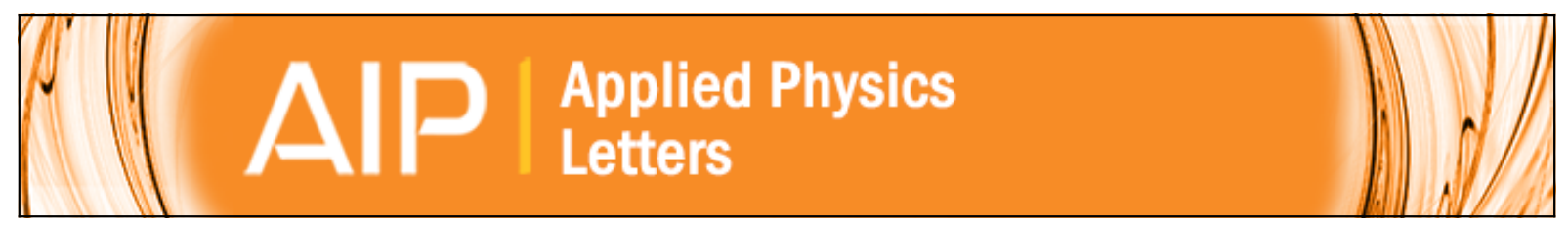

\title{
Electrical isolation in GaAs by light ion irradiation: The role of antisite defects
}

J. P. de Souza, I. Danilov, and H. Boudinov

Citation: Applied Physics Letters 68, 535 (1996); doi: 10.1063/1.116391

View online: http://dx.doi.org/10.1063/1.116391

View Table of Contents: http://scitation.aip.org/content/aip/journal/apl/68/4?ver=pdfcov

Published by the AIP Publishing

\section{Articles you may be interested in}

Defect engineering in GaAs using high energy light ion irradiation: Role of electronic energy loss

J. Appl. Phys. 109, 033701 (2011); 10.1063/1.3534003

Electrical isolation of p-type GaAs layers by ion irradiation

J. Appl. Phys. 91, 6585 (2002); 10.1063/1.1469693

Observation of $\mathrm{Ga}$ antisite defect in electron-irradiated semi-insulating $\mathrm{GaAs}$ by photoluminescence Appl. Phys. Lett. 61, 843 (1992); 10.1063/1.107763

Formation of AsGa antisite defects in electron-irradiated $\mathrm{GaAs}$

J. Appl. Phys. 58, 1041 (1985); 10.1063/1.336284

Electron spin resonance of AsGa antisite defects in fast neutron-irradiated GaAs

Appl. Phys. Lett. 40, 141 (1982); 10.1063/1.93016

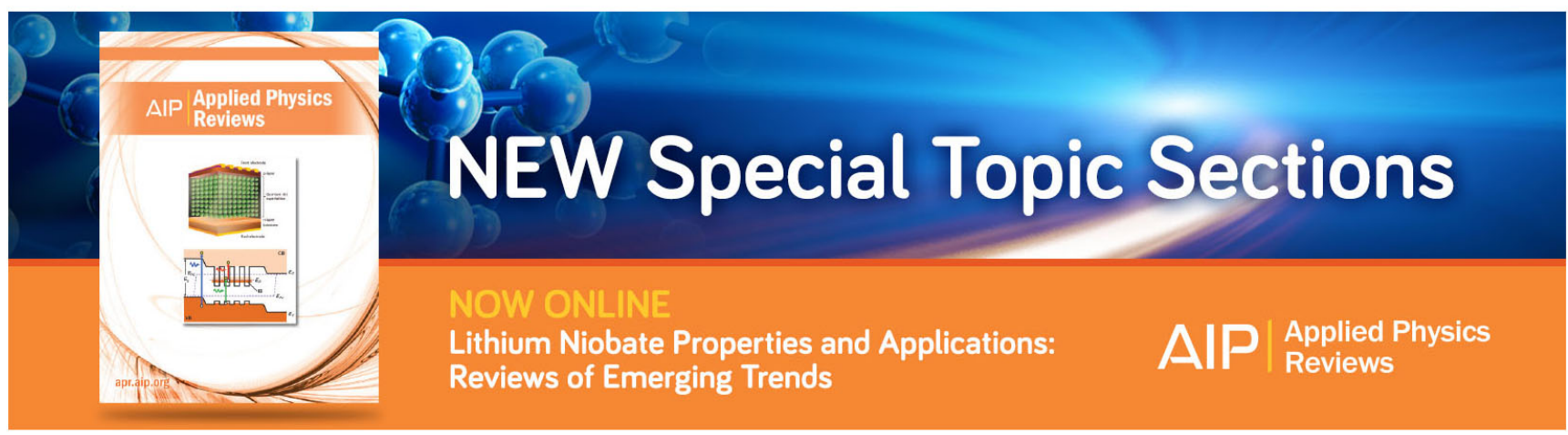




\title{
Electrical isolation in GaAs by light ion irradiation: The role of antisite defects
}

\author{
J. P. de Souza, ${ }^{\text {a) }}$ I. Danilov, and H. Boudinov \\ Instituto de Física, UFRGS, 91501-970 Porto Alegre, R.S., Brazil
}

(Received 9 October 1995; accepted for publication 14 November 1995)

\begin{abstract}
The evolution of the sheet resistance $\left(R_{s}\right)$ in $n$-type GaAs layers during ion irradiation was studied using light mass projectiles like proton, deuterium, and helium ions at various energies. For all the cases, at the beginning of the irradiation, $R_{s}$ increases with the accumulation of the dose. After reaching $\approx 10^{9} \Omega / \square, R_{s}$ saturates, forming a plateau. This plateau is succeeded by a decreasing of $R_{s}$ with the increase of the dose, denoting that conduction via damage-related mechanisms is taking place. The threshold dose to convert the conductive layer to a highly resistive one at room temperature or at $100{ }^{\circ} \mathrm{C}$ is found to scale with the inverse of the estimated number of displaced lattice atoms along the depth of the doped layer. Antisite defects formed by the replacement collisions are invoked to play the major role in isolation formation in GaAs by virtue of their lower sensitivity to dynamic annealing compared to other point defects. (C) 1996 American Institute of Physics. [S0003-6951(96)04904-1]
\end{abstract}

Ion implantation plays a fundamental role in the development of discrete devices and integrated circuits (ICs) in III-V compound semiconductor technology. The ion irradiation of the III-V materials presents a unique feature compared to that of $\mathrm{Si}$, since it can convert conductive layers to highly resistive ones. ${ }^{1-3}$ The masking of defined areas of the semiconductor surface with photoresist followed by irradiation with proper ion doses and energies constitutes an efficient and practical technique to isolate closely spaced devices. It is commonly assumed that the trapping of carriers at the irradiation damage or at deep centers associated to specific impurities used for irradiation (chemical isolation) are the mechanisms that promote electrical isolation.

The degree of electrical isolation after ion irradiation and low temperature annealing $\left(300-500{ }^{\circ} \mathrm{C}\right)$ is even superior than those provided by mesa structures. Commonly employed ions for isolation purposes include $\mathrm{B}^{+},{ }^{3} \mathrm{H}^{+}, 4 \mathrm{O}^{+}, 3,5$ etc. The choice of the ion depends on the thickness of the layer to be isolated and the required thermal stability of the isolation. ${ }^{1}$

Besides the fact that in semi-insulating (SI) GaAs wafers the devices are fabricated self-isolated by masked dopant implantations, the surface conditions in the passive regions may affect the isolation degree. One of these detrimental effects, called the backgating effect, occurs in complex GaAs ICs. It consists in the reduction of the drain-to-source current in field effect transistors by the negative biasing of a closely spaced $n$-type contact. It has been reported that the isolation by ion irradiation can significantly reduce the backgating effect. $^{4,6}$

Besides the fact that the isolation process is currently used in the industry, the physical processes involved with this process are not completely understood at present and reports of systematic investigations are lacking in the literature.

a)Electronic mail: souza@if.ufrgs.br
The present work deals with the formation of the electrical isolation in shallow Si-doped GaAs layers using light ion irradiation. The sheet resistance $\left(R_{s}\right)$ of the samples was monitored during irradiation with different ions at nominal room temperature (RT) or at $100{ }^{\circ} \mathrm{C}$. The ion dose required to increase $R_{s}$ to a maximum level was correlated with the estimated number of displaced lattice atoms $\left(N_{d}\right)$ in the doped layer.

Semi-insulated LEC GaAs wafers of (100) orientation were used throughout this work. The wafers were implanted to doses in the range of $1-7.5 \times 10^{13} \mathrm{~cm}^{-2}$ of ${ }^{29} \mathrm{Si}^{+}$or ${ }^{47} \mathrm{SiF}^{+}$at the energy of 30 or $50 \mathrm{keV}$, respectively. The thickness of the doped layer where the $\mathrm{Si}$ atom concentration exceeds $10^{16} \mathrm{~cm}^{-3}$ is $200 \mathrm{~nm}$, as measured by SIMS (not shown). The electrical activation of the implanted $\mathrm{Si}$ was performed by capless (Si proximity) rapid thermal annealing (RTA) in the temperature range of $825-925{ }^{\circ} \mathrm{C}$ for $10 \mathrm{~s}$. After RTA the samples were cleaved in pieces of $6 \times 3 \mathrm{~mm}^{2}$ for resistors devices or $6 \times 6 \mathrm{~mm}^{2}$ for van der Pauw devices. ${ }^{7}$ The ohmic contacts to the samples were provided by In applied to the GaAs surface after sintering at $\approx 200{ }^{\circ} \mathrm{C}$ for 2 min. The In layers form masks that prevent ion irradiation of the contact regions.

The devices were irradiated at RT with ${ }^{1} \mathrm{H}^{+}$at 50,100 , or $150 \mathrm{keV},{ }^{2} \mathrm{H}^{+}$at $40 \mathrm{keV}$ or ${ }^{4} \mathrm{He}^{+}$at $80 \mathrm{keV}$ in the dose range of $1 \times 10^{10}-1 \times 10^{16} \mathrm{~cm}^{-2}$ with ion current density in the range of $10 \mathrm{nA} / \mathrm{cm}^{2}$ to $0.5 \mu \mathrm{A} / \mathrm{cm}^{2}$. In a limited number of samples, the irradiation was conducted at $100{ }^{\circ} \mathrm{C}$. To minimize ion channeling the device surface normal was tilted by $7^{\circ}$ in respect to beam incidence direction. The energies were chosen in order to get the projected ranges at least two times deeper than the layer depth.

The $R_{s}$ values were measured after each dose step using a Keithley 617 electrometer without breaking the vacuum in the target chamber. The van der Pauw devices were irradiated to a single dose step and unloaded from the chamber for $R_{s}$ and Hall effect measurements.

Figure 1(a) presents the evolution of $R_{s}$ during ${ }^{1} \mathrm{H}^{+}$ir- 


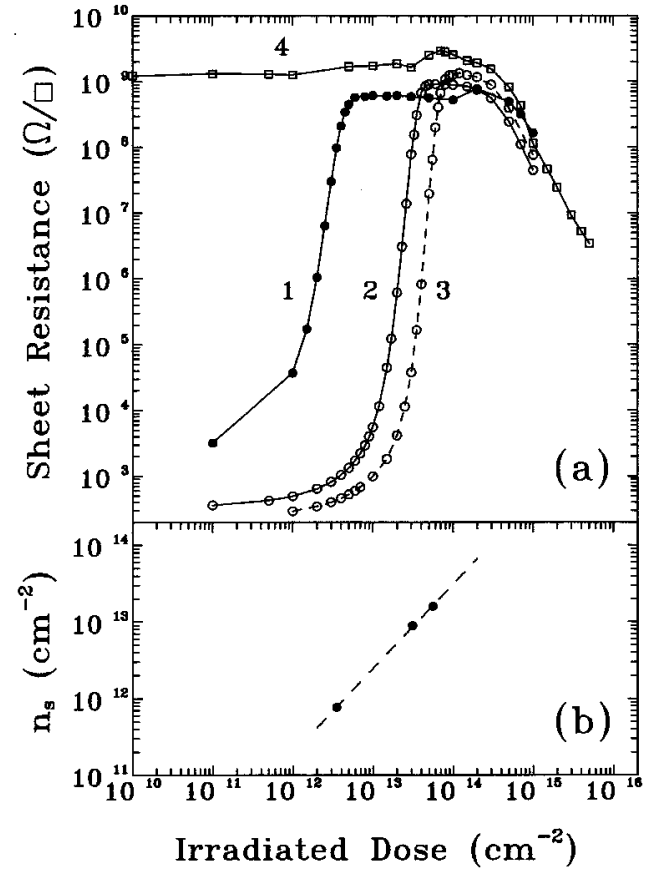

FIG. 1. (a) Dose dependence of the sheet resistance for $50 \mathrm{keV}{ }^{1} \mathrm{H}^{+}$irradiation at RT in samples having original sheet carrier concentration of 7.7 $\times 10^{11} \mathrm{~cm}^{-2}$ (curve 1), $8.9 \times 10^{12} \mathrm{~cm}^{-2}$ (curve 2), or $1.6 \times 10^{13} \mathrm{~cm}^{-2}$ (curve 3). Curve 4 represents the case of a SI GaAs sample submitted to an identical irradiation. (b) The original sheet carrier concentration of the doped samples are plotted vs the threshold doses obtained from data in (a). The straight line of slope 1.0 represents the best fitting to the data.

radiations at $50 \mathrm{keV}$ in resistors having original sheet carrier concentration $\left(n_{s}\right)$ of $7.7 \times 10^{11}$ (curve 1 ), $8.9 \times 10^{12}$ (curve 2), and $1.6 \times 10^{13} \mathrm{~cm}^{-2}$ (curve 3). The evolution of $R_{s}$ in an undoped SI sample submitted to ${ }^{1} \mathrm{H}^{+}$irradiation (at $50 \mathrm{keV}$ ) is also included in the figure (curve 4). At the beginning of the irradiation of the doped samples, $R_{s}$ increases with the increase of the dose. In the sample having the lower $n_{s}$ (see curve 1), $R_{s}$ presents a sharp increase of about five orders of magnitude $\left(\right.$ dose $\left.<5 \times 10^{12} \mathrm{~cm}^{-2}\right)$. Similar $R_{s}$ enhancement is observed in other doped samples (see curves 2 and 3 ). However, the dose interval, where the sharp enhancement of $R_{s}$ occurs, shifts to the higher dose regions proportionally with the value of the original $n_{s}$. Electrical measurements in van der Pauw devices revealed that $R_{s}$ increases due to mobility degradation and reduction of $n_{s}$ in consequence of carrier trapping. $R_{s}$ of the doped samples reach their highest levels $\left(\approx 10^{9} \Omega / \square\right)$ after proper doses (called hereafter threshold doses) have been accumulated. Continuing the dose accumulation, the $R_{s}$ values remain approximately constant forming plateaus that extend up to a dose of 2 $\times 10^{14} \mathrm{~cm}^{-2}$. The $R_{s}$ levels in the plateaus in Fig. 1(a) are of the same order of magnitude of that the SI sample (curve 4) and hence correspond to the lack of mobile carriers in the doped layers.

The decreasing of $R_{s}$ for doses beyond $2 \times 10^{14} \mathrm{~cm}^{-2}$ has been explained by the onset of damage-related conduction mechanisms. ${ }^{1}$ Since this conduction starts to manifest at a similar dose in the four samples, one can presume that the

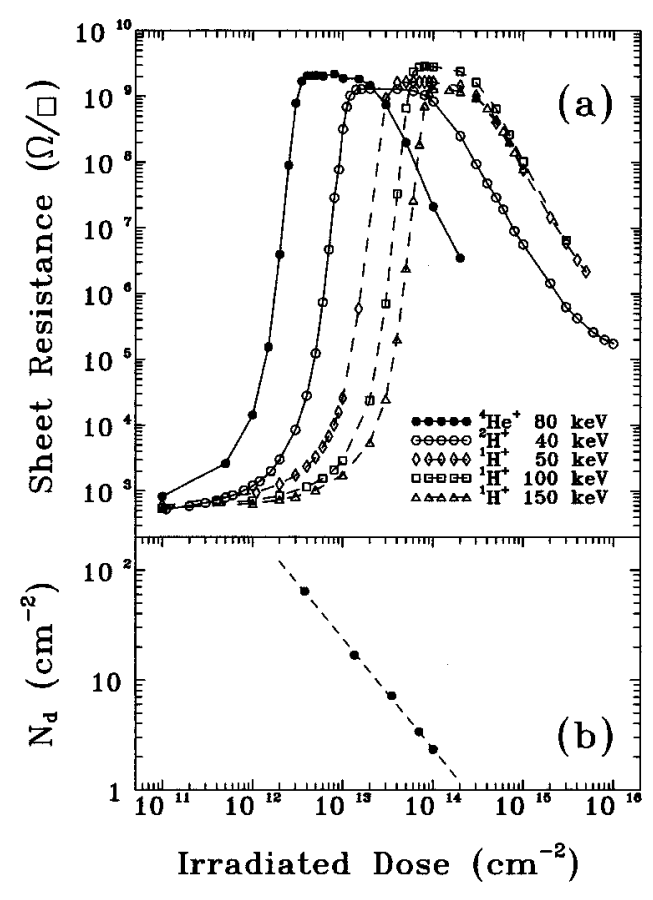

FIG. 2. (a) Dose dependence of the sheet resistance in identical samples for irradiation at RT with ${ }^{1} \mathrm{H}^{+}$at 50,100 , or $150 \mathrm{keV},{ }^{2} \mathrm{H}^{+}$at $40 \mathrm{keV}$ or ${ }^{4} \mathrm{He}^{+}$at $80 \mathrm{keV}$. (b) The estimated numbers of displaced lattice atoms $\left(N_{d}\right)$ are plotted vs the threshold doses obtained from data in (a). The straight line of slope -1.0 represents the best fitting to the data.

concentration of accumulated damage is the main parameter governing this kind of conductivity.

In Fig. 1(b) the ${ }^{1} \mathrm{H}^{+}$threshold doses [taken from data in Fig. 1(a)] are plotted versus the original values of $n_{s}$. For such an $R_{s}$ value, the carrier concentration in the doped layer is negligible. The fitting to the data in Fig. 1(b) with a straight line of slope 1.0 suggests that the carrier trapping scales with the irradiated dose and thus with deposited energy in the doped layer. From the data in Fig. 1(b) one can estimate that an average number of 4.7 protons of $50 \mathrm{keV}$ energy is required for a single carrier removal.

Figure 2(a) shows the evolution of $R_{s}$ in identical resistors $\left(n_{s}=7 \times 10^{12} \mathrm{~cm}^{-2}\right)$ irradiated with ${ }^{1} \mathrm{H}^{+}$at 50,100 , and $150 \mathrm{keV},{ }^{2} \mathrm{H}^{+}$at $40 \mathrm{keV}$ or ${ }^{4} \mathrm{He}^{+}$at $80 \mathrm{keV}$. It is clearly apparent the progressive shift of the curves towards lower dose region with the decrease of the ion energy or the increase of the ion mass.

In Fig. 2(b) are plotted the estimated number of lattice atom displacements produced per individual ion in the doped layer (according to TRIM simulations ${ }^{8}$ ) versus the threshold doses. Lattice atom displacement energies of $9 \mathrm{eV}$ for $\mathrm{Ga}$ and $10 \mathrm{eV}$ for As were adopted in the simulations. ${ }^{9}$

The fitting to the data in Fig. 2(b) with a straight line of slope -1.0 demonstrates that the carrier removal processes scale with the inverse of the estimated $N_{d}$.

The evolution of $R_{s}$ with the $\mathrm{He}^{+}$dose at $80 \mathrm{keV}$ is compared in Fig. 3 for irradiations performed at RT and $100{ }^{\circ} \mathrm{C}$. In the latter case, the $R_{s}$ measurements were performed without cooling the sample, i.e., at $100{ }^{\circ} \mathrm{C}$. The lower level of the plateau corresponding to the hot irradiation is 


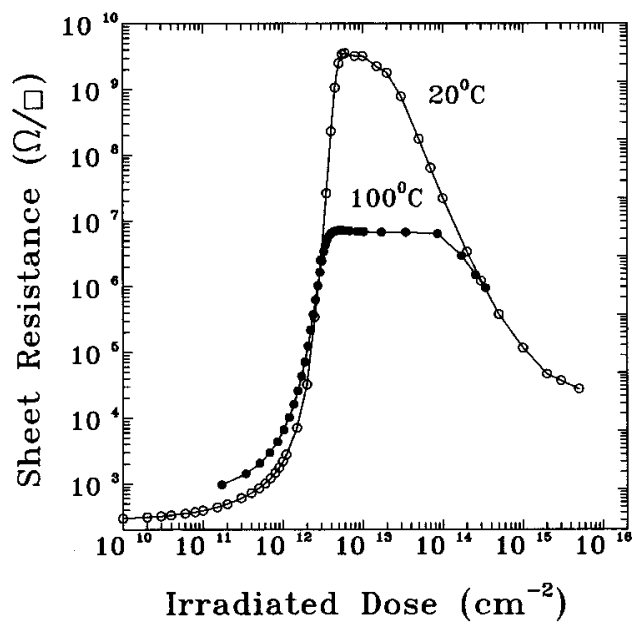

FIG. 3. Dose dependence of the sheet resistance for $80 \mathrm{keV}^{4} \mathrm{He}^{+}$irradiation at RT $\left(20^{\circ} \mathrm{C}\right)$ or $100{ }^{\circ} \mathrm{C}$.

caused by the thermal increasing of the GaAs conductivity. It is interesting to note that quite similar $\mathrm{He}^{+}$threshold doses $\left(4-5 \times 10^{12} \mathrm{~cm}^{-2}\right)$ were found for irradiations conducted at $\mathrm{RT}$ and at $100{ }^{\circ} \mathrm{C}$. The same temperature dependence of the threshold dose is obtained for ${ }^{1} \mathrm{H}^{+}$irradiations (not shown). These data are in apparent contrast with the lower damage accumulation that occurs by virtue of the enhanced dynamic annealing during the irradiations at $100{ }^{\circ} \mathrm{C}$. Consequently, the carrier trapping should be associated with irradiation defects of weak sensitivity to dynamic annealing. This fact strongly suggests that they are presumably antisite defects $\left(\mathrm{Ga}_{\mathrm{As}}\right.$ and $\left.\mathrm{As}_{\mathrm{Ga}}\right)$ that are formed at the replacement collisions in the doped layer. Very likely the structures of the carrier trapping sites may include antisite-related defect complexes as well.
According to $\mathrm{TRIM}^{8}$ simulations, the numbers of replacement collisions per income ion taking place in the doped layer for irradiations conducted with $150 \mathrm{keV}{ }^{1} \mathrm{H}^{+}, 100 \mathrm{keV}$ ${ }^{1} \mathrm{H}^{+}, 50 \mathrm{keV}{ }^{1} \mathrm{H}^{+}, 40 \mathrm{keV}{ }^{2} \mathrm{H}^{+}$, and $80 \mathrm{keV}{ }^{4} \mathrm{He}^{+}$are 0.58 , $0.84,1.84,4.00$, and 16.7 , respectively. We have to consider that only one half of the replacement collisions form antisite defects. Thus, dividing the numbers of replacement collisions by two and multiplying by the threshold dose [from Fig. 2(b)], one obtains values of the same order of magnitude of the original $n_{s}$.

In summary, we studied the effects of light ion irradiation on the sheet resistance of $n$-type GaAs layers. The threshold dose to convert the $n$ layer to a highly resistive one was found to closely correlate with the estimated number of lattice atom displacements along the depth of the doped layer. The threshold doses are quite similar for irradiations conducted at RT or at $100{ }^{\circ} \mathrm{C}$, in spite of the enhanced dynamic annealing in the last case. Antisite defects originating at the replacement collisions and/or their related defect complexes are considered to be the carrier trapping centers, by virtue of their low sensitivity to dynamic annealing.

This work was partially supported by Conselho Nacional de Pesquisas $(\mathrm{CNPq})$ and Financiadora de Estudos e Projetos (Finep).

${ }^{1}$ S. J. Pearton, Mater. Sci. Rep. 4, 313 (1990).

${ }^{2}$ D. V. Morgan, F. H. Eisen, and A. Ezis, IEE Proc. 128, 109 (1981).

${ }^{3}$ K. T. Short and S. J. Pearton, J. Electrochem. Soc. 135, 2835 (1988).

${ }^{4}$ D. C. D'Avanzo, IEEE Trans. Electron Devices ED-29, 1051 (1982).

${ }^{5}$ P. N. Favennec, J. Appl. Phys. 47, 2532 (1976).

${ }^{6}$ J. P. de Souza and D. K. Sadana, Mater. Res. Soc. Symp. Proc. 240, 887 (1991).

${ }^{7}$ L. J. Van der Pauw, Phillips Res. Rep. 13, 1 (1958).

${ }^{8}$ J. F. Ziegler, J. P. Biersack, and U. Littmark, The Stopping and Range of Ions in Solids (Pergamon, Oxford, 1985), Vol. 1.

${ }^{9}$ J. W. Corbett and J. C. Bourgoin, in Point Defects in Solids, edited by J. H. Crawford and L. M. Slifkin (Plenum, New York, 1975), p. 118. 